\title{
Atomic structure factors from a molecular integral equation theory: An application to homonuclear diatomic fluids
}

Cite as: J. Chem. Phys. 102, 2092 (1995); https://doi.org/10.1063/1.468730

Submitted: 08 August 1994 . Accepted: 26 October 1994 . Published Online: 31 August 1998

C. Martín, M. Lombardero, M. Alvarez, and E. Lomba

ARTICLES YOU MAY BE INTERESTED IN

Potentials of mean force of simple ions in ambient aqueous solution. I. Three-dimensional reference interaction site model approach

The Journal of Chemical Physics 112, 10391 (2000); https://doi.org/10.1063/1.481676

Statistical Mechanics of Fluid Mixtures

The Journal of Chemical Physics 3, 300 (1935); https://doi.org/10.1063/1.1749657

Density functional theory of nonuniform polyatomic systems. I. General formulation

The Journal of Chemical Physics 85, 5971 (1986); https://doi.org/10.1063/1.451510
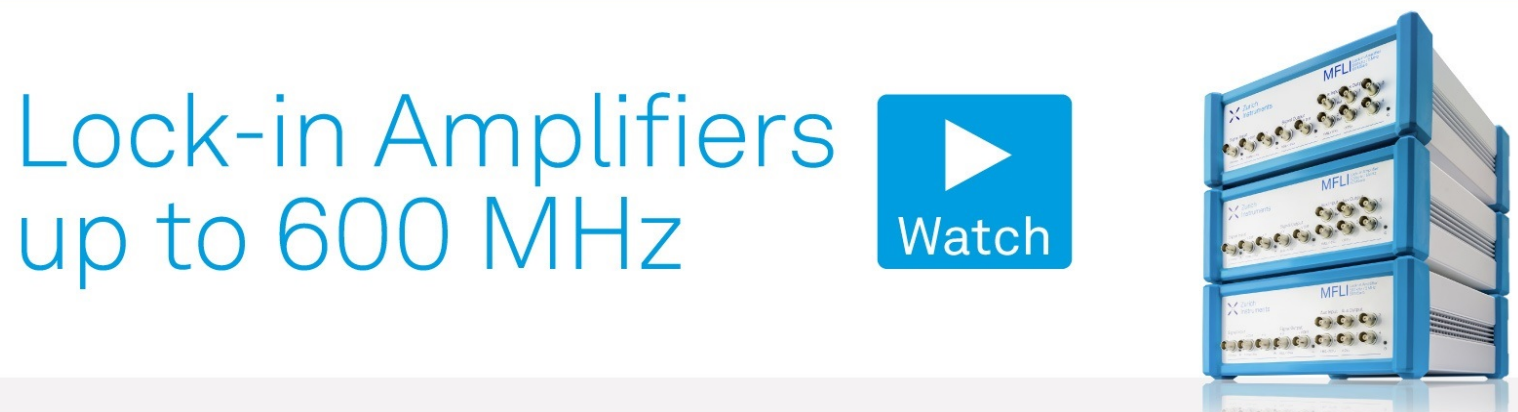

J. Chem. Phys. 102, 2092 (1995); https://doi.org/10.1063/1.468730 


\title{
Atomic structure factors from a molecular integral equation theory: An application to homonuclear diatomic fluids
}

\author{
C. Martín and M. Lombardero \\ Instituto de Química Física Rocasolano, CSIC, Serrano 119, E-28006 Madrid, \\ and Departamento Química Física I, U. Complutense, E-28040 Madrid, Spain \\ M. Alvarez and E. Lomba \\ Instituto de Química Física Rocasolano, CSIC, Serrano 119, E-28006 Madrid, Spain
}

(Received 8 August 1994; accepted 26 October 1994)

\begin{abstract}
We present a novel approach for the theoretical determination of atomic structure factors (or site-site distribution functions) based on the calculation of the molecular pair distribution function by integral equations (reference hypernetted chain approximation). The results are compared with experimental structure factors and computer simulation results for homonuclear diatomic fluids $\left(\mathrm{N}_{2}, \mathrm{Cl}_{2}\right.$ and $\left.\mathrm{Br}_{2}\right)$ which are modeled by means of two center Lennard-Jones potentials. The proposed method leads to a surprisingly good agreement with experimental data, within the obvious limitations that stem from intrinsic inadequacies of the model interaction potential. Comparison with RISM integral equation results evidences the superiority of the molecular integral equation approach. () 1995 American Institute of Physics.
\end{abstract}

\section{INTRODUCTION}

The theoretical analysis of fluids composed of diatomic molecules, modeled by two-center Lennard-Jones (2CLJ) (with or without multipoles) has reached a considerable development within the framework of molecular integral equation theories, in particular since the advent of the reference hypernetted chain equation (RHNC) ${ }^{1}$ and its extension to fluids of nonspherically shaped molecules. ${ }^{2}$ In a series of recent works ${ }^{3-7}$ it has been shown that the use of parametrized hard-dumbbell reference bridge functions determined in the modified Verlet's approximation, ${ }^{8}$ is an excellent tool for accurately describing both the thermodynamics and the microscopic structure of these molecular fluids. This approach will be denoted hereafter by RHNC-VM in keeping with Refs. 3-7. There is, however, one aspect that has remained partially unexplored so far, and is the determination of the site-site (atom-atom) distribution functions. These are quantities of extreme interest, being the only microscopic structural properties that can be directly extracted from diffraction experiments (more properly the atom-atom structure factors). Up to now, the only available theoretical approaches in this context were based on RISM-type (reference interaction site model) integral equations, ${ }^{9,10}$ which have fundamental limitations, in particular due to the lack of a systematic way to improve their closure relation. Therefore, we decided to probe the ability of molecular integral equation theories to provide accurate site-site distribution functions. As will be seen later, the calculation of these site-site correlation functions is straightforward in the case of homonuclear diatomic fluids, once a general algorithm to treat heteronuclear diatomics is available. ${ }^{7}$

As a test of our method we have chosen three models of 2CLJ systems that represent liquid nitrogen, ${ }^{11}$ liquid chlorine ${ }^{12}$ and liquid bromine, ${ }^{13}$ systems for which there is a considerable amount of experimental structural information (see Refs. 14 and 15 for $\mathrm{N}_{2}$, Refs. 16 and 17 for $\mathrm{Cl}_{2}$, and Refs. 18-20 for $\mathrm{Br}_{2}$ ). For these systems we have performed extensive Monte Carlo simulations, in order to compare the simulated site-site distributions functions with results from the RHNC-VM equation and RISM integral equation data, in this latter instance using both Percus-Yevick (PY) and HNC-type closures.

Our theoretical structure factors will also be compared with x-ray and neutron diffraction data from Refs. 14-20, which will make even more evident the clear superiority of the molecular integral equation approach vs site-site integral equation theories.

The rest of the paper can be sketched as follows. In Section II, we will briefly summarize the RHNC-VM theory in the way it has been used here. The essential equations of the RISM integral equation are also collected therein. In Section III, we will outline the procedures required to express the experimental results in a way in which they can be directly compared with the integral equation output. Finally, in Sec. IV we present and comment the prime results of this work.

\section{INTEGRAL EQUATION THEORY: MOLECULAR VERSUS SITE-SITE APPROACH}

The molecular Ornstein-Zernike equation for fluids composed of linear particles reads

$$
\gamma(12)=\frac{\rho}{4 \pi} \int c(13)[c(32)+\gamma(32)] d \mathbf{3},
$$

where $\gamma(12)=g(12)-1-c(12)$ is the indirect correlation function, $c(12)$ and $g(12)$ are the direct correlation and pair distribution functions, respectively, and $\rho$ is the molecular number density.

The RHNC closure can be written as

$$
c(12)=\exp \left[-\beta u(12)+\gamma(12)-B_{0}(12)\right]-\gamma(12)-1,
$$

where $\beta=1 / k T, u(12)$ is the interaction, in this case twocenter $\mathrm{LJ}$ potential, and $B_{0}(12)$ is the bridge function of a 
TABLE I. Lennard-Jones potential parameters for the systems considered in this work.

\begin{tabular}{lrll}
\hline \hline & $\epsilon / K$ & $\sigma(\AA)$ & $L(\AA)$ \\
\hline $\mathrm{N}_{2}$ & 37.3 & 3.310 & 1.090 \\
$\mathrm{Cl}_{2}$ & 178.3 & 3.332 & 2.10 \\
$\mathrm{Br}_{2}$ & 316.1 & 3.610 & 2.27 \\
\hline \hline
\end{tabular}

given reference system. The standard procedure to solve this Eq. (2) involves the expansion of the correlation function in spherical harmonics, i.e.,

$$
g\left(R, \omega_{1}, \omega_{2}\right)=4 \pi \sum_{k l m} g_{k l m}(R) Y_{k m}\left(\omega_{1}\right) Y_{l m}\left(\omega_{2}\right)
$$

which, by use of the orthogonality properties of the angular basis functions, transforms the convolution of Eq. (1) into a matrix equation in Fourier space. ${ }^{2}$

It is precisely through the expansion (3) that the relation between the molecular pair distribution function and the sitesite distribution functions can easily be established. If molecular orientations are defined in an axial reference frame in which the $z$-axis connects two sites (denoted by $\alpha$ ) of two molecules (instead of the usual center-of-mass to center-ofmass $z$-axis), then the first term in the expansion is simply the $\alpha \alpha$-site-site function, i.e.,

$$
g_{000}(R)=g_{\alpha \alpha}(r),
$$

where $r$ is the site-site distance and $R=r$ according to our definition of the reference frame.

It is crucial to bear in mind that this relation will only hold if the axial reference frame is defined as indicated above. More generally one could write ${ }^{21}$

$$
\begin{aligned}
g_{\alpha \beta}(\mathbf{r})= & \frac{1}{4 \pi^{2}} \int d \mathbf{R} d \omega_{1} d \omega_{2} g\left(\mathbf{R}, \omega_{1}, \omega_{2}\right) \\
& \times \delta\left(\mathbf{R}+\mathbf{l}_{2 \beta}\left(\omega_{2}\right)-\mathbf{l}_{1 \alpha}\left(\omega_{1}\right)-\mathbf{r}\right)
\end{aligned}
$$

with $\mathbf{l}_{i \alpha}$ denoting the vector displacement of site $\alpha$ from molecular center $i$. One might try to perform this integration in a general way, however this turns out be a cumbersome task due to the $\delta$-function constraints on the integration variables. On the contrary, if the reference frame is defined as mentioned before, then $l_{2 \beta}=l_{1 \alpha}=0$ in a homonuclear diatomic, hence integration over $\mathbf{R}$ yields a simple $\delta$-function contribution at $\mathbf{R}=\mathbf{r}$

$$
g_{\alpha \beta}(r)=g_{000}(r)=\frac{1}{4 \pi^{2}} \int d \omega_{1} d \omega_{2} g\left(r, \omega_{1}, \omega_{2}\right)
$$

with a nonconstrained integration over orientations.

Note that the proposed procedure is only valid for homonuclear diatomics, and in a more general case one must resort to Eq. (5). Work on this general treatment is already in progress. Finally, it has also to be stressed that, although the system is homonuclear (with all the symmetry simplifications this implies), the required choice of reference system breaks the molecular symmetry with respect to the reference frame, and therefore, the expansion in spherical harmonics must include all components appearing in a heteronuclear
TABLE II. Thermodynamic states of the systems considered in this work.

\begin{tabular}{lcccccc}
\hline \hline & \multicolumn{2}{c}{$\mathrm{N}_{2}$} & \multicolumn{2}{c}{$\mathrm{Cl}_{2}$} & \multicolumn{2}{c}{$\mathrm{Br}_{2}$} \\
\hline$T(\mathrm{~K})$ & 66.4 & 77.0 & 200.0 & 290.0 & 293.0 & 473.0 \\
$\rho\left(\mathrm{g} / \mathrm{cm}^{3}\right)$ & 0.8541 & 0.808 & 1.660 & 1.4188 & 3.1182 & 2.4413 \\
$T^{*}$ & 1.780 & 2.064 & 1.122 & 1.626 & 0.927 & 1.496 \\
$\rho^{*}$ & 0.6658 & 0.6299 & 0.5216 & 0.4458 & 0.5528 & 0.4328 \\
\hline \hline
\end{tabular}

system, i.e., no longer coefficients with even indices will be the only contribution to $g(12)$. When defining the reference system, however, we will use the expression proposed in Refs. 4 and 5 for homonuclear systems, namely

$$
B_{0}(12)=\frac{1}{2} \frac{\gamma_{0}^{2}(12)}{1+\xi\left(T^{*}, \rho^{*}\right) \gamma_{0}(12)}
$$

with $\gamma_{0}(12)$ being the indirect correlation function of a reference homonuclear hard diatomic fluid, in which the constituent hard sphere diameter is given by

$$
d^{*}=d_{a}^{*}\left(1+\frac{3}{2} L^{*}-\frac{1}{2} L^{*^{3}}\right)^{1 / 3}
$$

with $d_{a}^{*}=\alpha[25-\ln (k T / \epsilon)]\left(25+\ln \rho \sigma^{3}\right) \quad$ (where $\alpha=1.62$ $\times 10^{-3}$ and $\epsilon, \sigma$ are the Lennard-Jones potential parameters). Also in Eq. (6), $\xi\left(T^{*}, \rho^{*}\right)=1-\frac{1}{3} \rho \sigma^{3} d^{*^{3}}\left(T^{*}, \rho^{*}\right)$ and $L^{*}=L / \sigma$ is the diatomic reduced bond length. Even if the reference system is homonuclear, for consistency the $\gamma_{0}(12)$ will have to be determined in a reference frame with the $z$-axis along the line connecting the atomic sites, and hence, once again no symmetry simplifications apply (except those which hold for all linear molecules).

We can now briefly recall the essentials of the site-site integral equation approach. With the atom-atom correlations split into intra- and intermolecular parts, and defining the intramolecular correlation components for a diatomic of fixed bond length $L$ as

$$
\omega_{\alpha \beta}(r)=\delta_{\alpha \beta} \delta(\mathbf{r})+\left(1-\delta_{\alpha \beta}\right) \delta(\mathbf{r}-L)
$$

one then has an Ornstein-Zernike relation connecting the intermolecular site-site direct and total correlation functions, that in Fourier space and in matrix notation reads

$$
\tilde{\mathbf{h}}(Q)=\tilde{\omega}(Q) \tilde{\mathbf{c}}(Q) \tilde{\omega}(Q)+\rho \tilde{\omega}(Q) \tilde{\mathbf{c}}(Q) \tilde{\mathbf{h}}(Q) .
$$

This site-site equation needs now a corresponding closure, which is usually defined by direct generalization of standard closures valid for simple fluids and mixtures, as the PY or HNC approximations. Then one would have

$$
h_{\alpha \beta}(r)=\exp \left[-\beta u_{\alpha \beta}(r)+h_{\alpha \beta}(r)-c_{\alpha \beta}(r)\right]-1
$$

in the $\mathrm{HNC}$, or

$$
c_{\alpha \beta}(r)=\left[\exp \left\{-\beta u_{\alpha \beta}(r)\right\}-1\right]\left[1+h_{\alpha \beta}(r)-c_{\alpha \beta}(r)\right]
$$

in the PY approximation.

Note that there is not a corresponding generalization of the RHNC closure Eq. (2), since Eqs. (11) and (12) are simple extensions to the site-site formalism of the standard diagrammatic analysis of the correlation functions, which does not apply in the same simple way as does for mixtures. Chandler's et al. reformulation ${ }^{22}$ to make the theory proper 


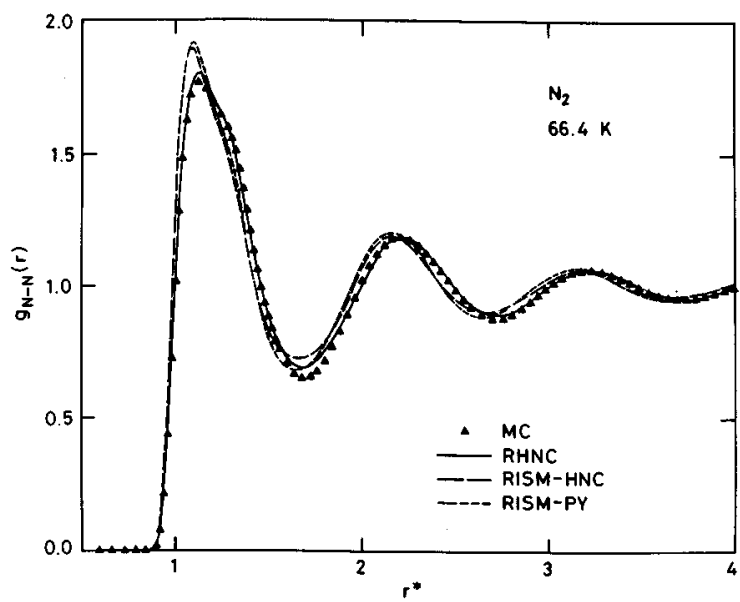

FIG. 1. N-N distribution function for liquid $\mathrm{N}_{2}$ at $T=66.4 \mathrm{~K}$. RHNC and RISM-HNC, RISM-PY vs MC simulations.

hardly improves the results and will therefore not be considered here. As expressed by Eqs. (10) and (11) or (12), the RISM theory is not "diagrammatically proper" and well established results for simple fluids must be translated into RISM language with some care. However, empirical evidence suggests that also here the use of the RISM equation with a HNC closure is best suited to systems with long range interactions, ${ }^{23}$ being the PY closure more adequate for short range repulsive potentials. ${ }^{24}$

\section{DATA REDUCTION}

In order to extract the experimental structural information to be used as benchmark of our results, the diffraction data taken from the literature (Refs. 14-20) (where they are presented in different ways) have been mostly treated following a so-called data reduction process. A sketched description of this process is given bellow for the reader convenience.

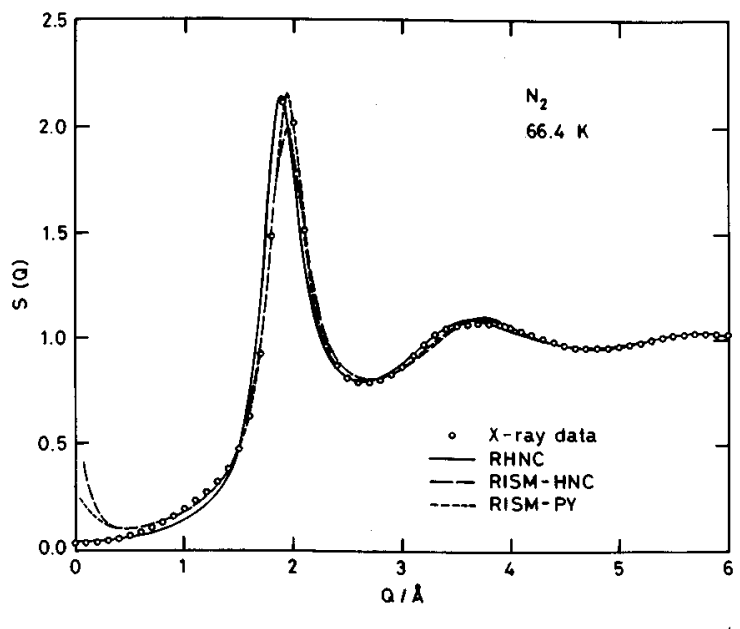

FIG. 2. N-N structure factor for $\mathrm{N}_{2}$ at $T=66.4 \mathrm{~K}$. RHNC, RISM-HNC, RISM-PY vs x-ray data from Ref. 14.

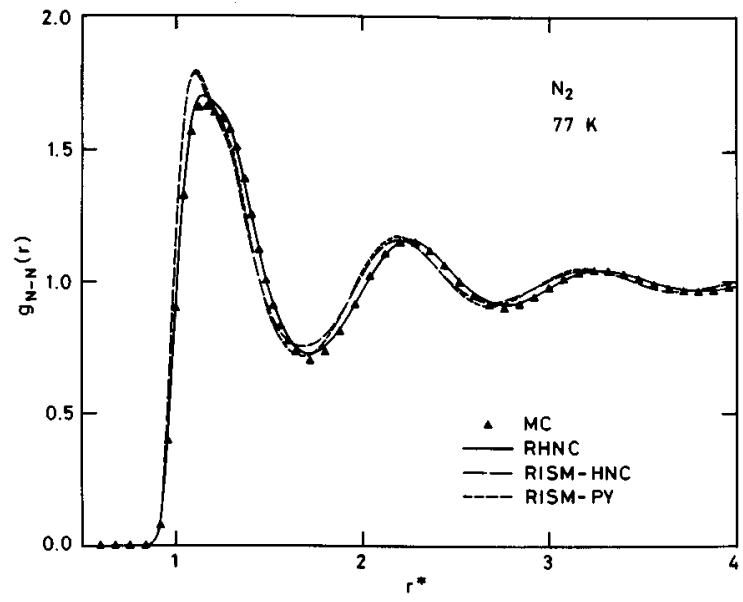

FIG. 3. Same as Fig. 1 but for $T=77 \mathrm{~K}$.

In the case of thermal neutron diffraction experiments, the observed magnitude (standard corrections aside) is essentially the differential cross section, $d \sigma / d \Omega$, resulting from two contributions,

$$
\left.\frac{d \sigma}{d \Omega}\right|_{\mathrm{obs}}=\left.\frac{d \sigma}{d \Omega}\right|_{\mathrm{inc}}+\left.\frac{d \sigma}{d \Omega}\right|_{\mathrm{coh}},
$$

where inc and coh refer to incoherent and coherent contribution, respectively. The structural information of the system under study is contained within the coherent term. In the case of molecular liquids, this contribution is related to the molecular static structure factor, $S_{m}(Q)$, as follows:

$$
\left.\frac{d \sigma}{d \Omega}\right|_{\text {coh }}=S_{m}(Q) /\left[\sum_{\alpha}^{n} b_{\alpha}\right]^{2}
$$

with $n$ being the number of atoms in the molecule, $b_{\alpha}$ the scattering lengths, and $Q$ the momentum transfer. $S_{m}(Q)$ can be expressed in terms of

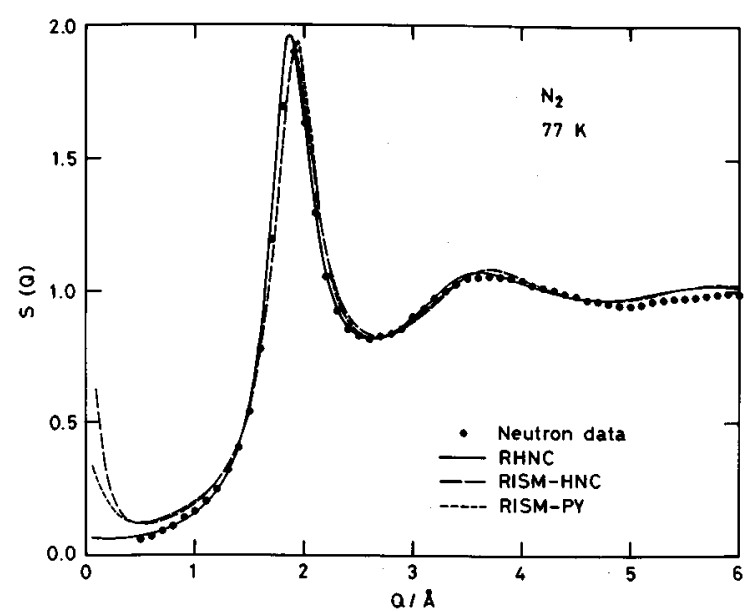

FIG. 4. Same as Fig. 2 but for $T=77 \mathrm{~K}$. Experimental data are neutron diffraction data from Ref. 15. 


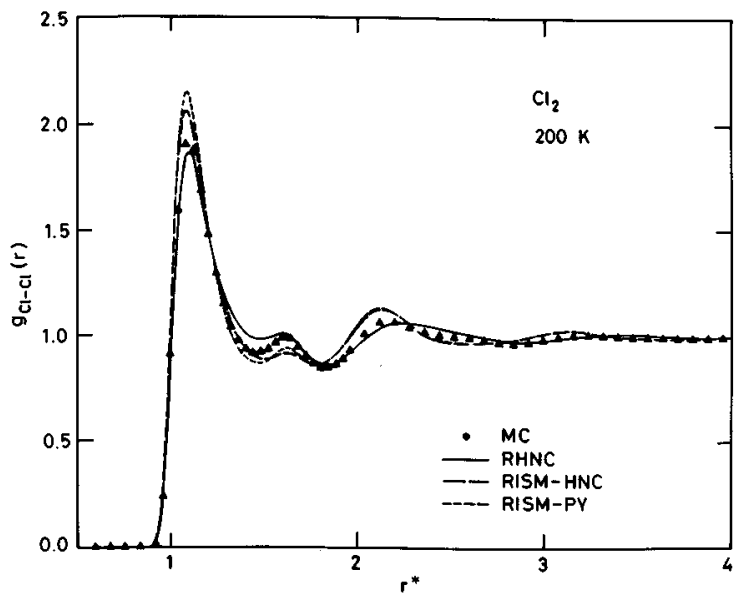

FIG. 5. Cl-Cl distribution function for liquid $\mathrm{Cl}_{2}$ at $T=200 \mathrm{~K}$. RHNC and RISM-HNC, RISM-PY vs MC simulations.

$$
S_{m}(Q)=f_{1}(Q)+D_{m}(Q) .
$$

The form factor, $f_{1}(Q)$, accounts for the intramolecular contribution and in the case of a homonuclear diatomic molecule is mainly given by,

$$
f_{1}(Q)=\frac{1}{2}\left(1+\frac{\sin (Q L)}{Q L}\right)
$$

with $L$ being the bond length. Obviously $f_{1}(Q)$ is essentially the Fourier transform of $w_{\alpha \beta}(r)$. On the other hand, the intermolecular part is related to the atomic pair distribution function, $g_{\alpha \beta}(r)$, as follows:

$$
D_{m}(Q)=\rho \int\left(g_{\alpha \beta}(r)-1\right) \exp (-i Q \boldsymbol{r}) d \boldsymbol{r}=S_{\alpha \beta}(Q)-1,
$$

where $S_{\alpha \beta}(Q)$ is the atomic structure factor, and $\alpha, \beta$ refer again to any pair of sites (atoms) located at different molecules. Therefore eventually all the data were converted to $S_{\alpha \beta}(Q)$.

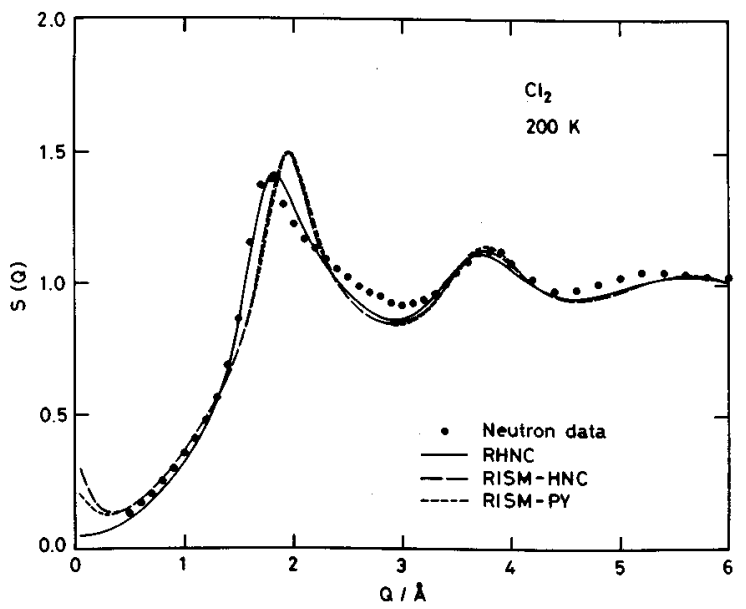

FIG. 6. $\mathrm{Cl}-\mathrm{Cl}$ structure factor for $\mathrm{Cl}_{2}$ at $T=200 \mathrm{~K}$. RHNC, RISM-HNC, RISM-PY vs experimental data from Ref. 16.

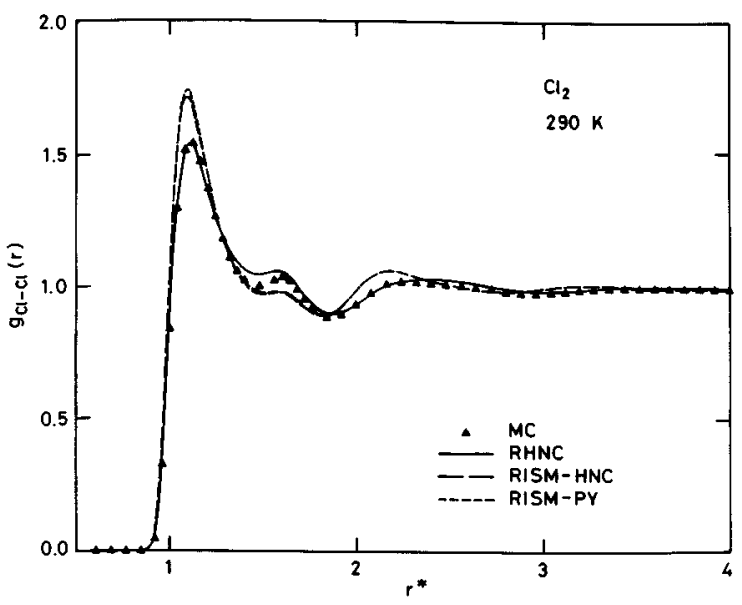

FIG. 7. Same as Fig. 5 but for $T=290 \mathrm{~K}$.

In summary, the total scattering intensity at a given momentum transfer is proportional to $S_{\alpha \beta}(Q)$ provided the intramolecular part and the incoherent contribution have been removed. The procedure is quite similar for x-ray diffraction data.

\section{RESULTS}

We have considered three different two-center LennardJones models to represent respectively $\mathrm{N}_{2}, \mathrm{Cl}_{2}$ and $\mathrm{Br}_{2}$, and for each of three models two thermodynamic states have been studied. Values of the Lennard-Jones parameters for each model can be found in Table I. Calculations were performed for the thermodynamic states summarized in Table II.

RISM equations were solved both with PY and HNC closures, except at the lowest $T^{*}$ for $\mathrm{Br}_{2}$, for which only RISM-HNC converged. The molecular integral equation was solved in the RHNC-VM approximation using 35 coefficients (i.e. $g_{k l m}$, with $k, l, m \leqslant 4$ ). The results for $\mathrm{N}_{2}$ are shown on Figs. 1-2 for $77 \mathrm{~K}$ and Figs. 3 and 4 for $66.4 \mathrm{~K}$. Figures 1 and 3 show a comparison of the $\mathrm{N}-\mathrm{N}$ distribution

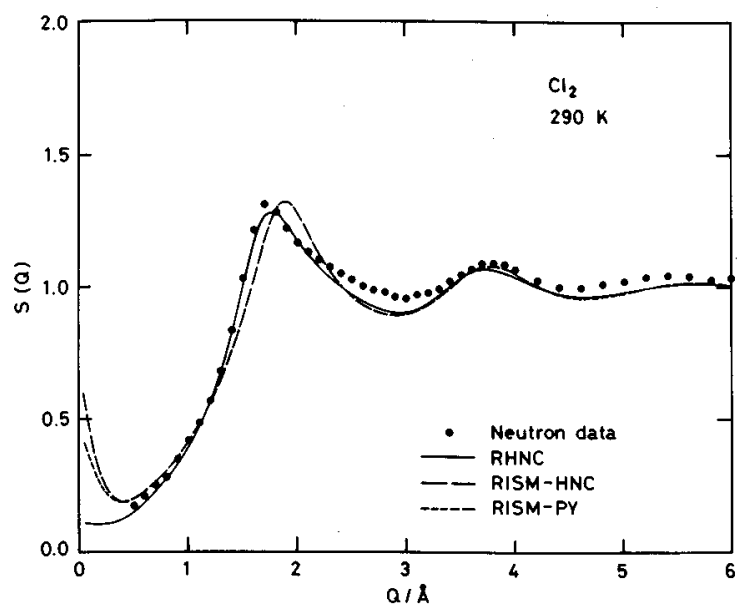

FIG. 8. Same as Fig. 6 but for $T=290$ K. Experimental data are neutron diffraction data from Ref. 16. 


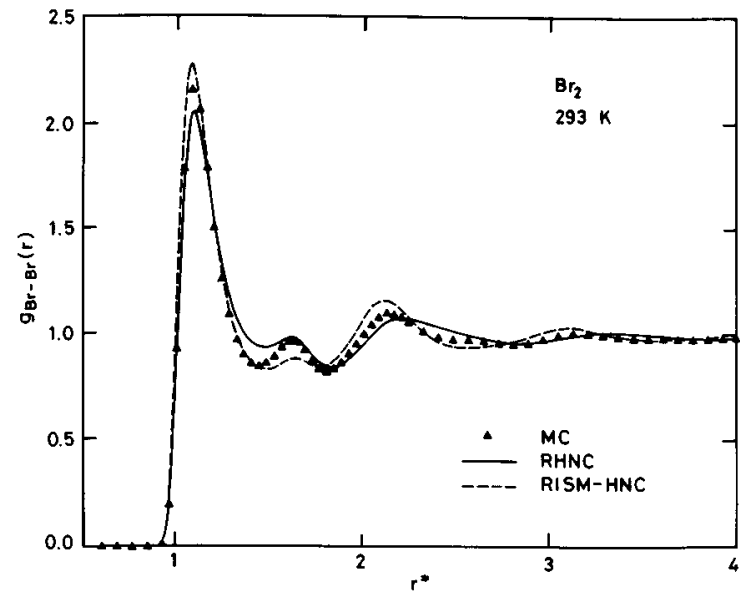

FIG. 9. $\mathrm{Br}-\mathrm{Br}$ distribution function for liquid $\mathrm{Br}_{2}$ at $T=293 \mathrm{~K}$. RHNC and RISM-HNC vs MC simulations.

function from the molecular RHNC-VM approximation and RISM-HNC and RISM-PY vs MC results. We observe that RISM results are clearly out of phase and miss the first peak at both temperatures as well. In Figures 2 and 4 one can compare the theoretical structure factors with neutron ${ }^{15}$ and $x$-ray ${ }^{14}$ diffraction. The overall superiority of the molecular RHNC is clear. In particular is interesting the low $Q$ behavior which is not correctly reproduced by the site-site integral equations.

Results for $\mathrm{Cl}_{2}$ are presented in Figs. 5 and 6 for $T=200 \mathrm{~K}$ and Figs. 7 and 8 for $T=290 \mathrm{~K}$. Though the agreement between RHNC theory and simulation is not so impressive as for liquid $\mathrm{N}_{2}$, it can still be deemed good, and far better than that of RISM equations. In particular, once again the discrepancies for the low $Q$ structure factor are much larger for the latter. The fact that the disagreement between computer simulation and RHNC theory is larger for $\mathrm{Cl}_{2}$ than for $\mathrm{N}_{2}$ might well be connected with the larger elongation of the molecular model $\left(L_{\mathrm{Cl}_{2}}^{*} \approx 2 L_{\mathrm{N}_{2}}^{*}\right)$. One must

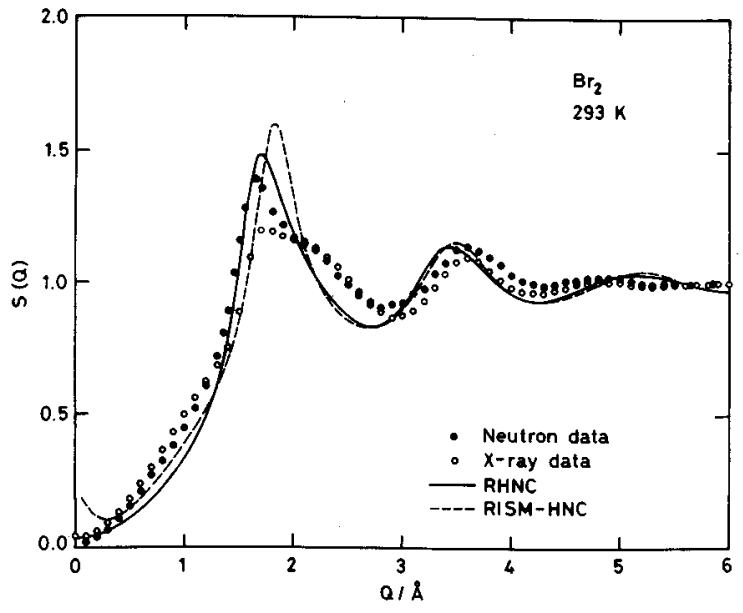

FIG. 10. $\mathrm{Br}-\mathrm{Br}$ structure factor for $\mathrm{Br}_{2}$ at $T=293 \mathrm{~K}$. RHNC and RISMHNC vs both x-ray (Ref. 18) and neutron diffraction data (Ref. 20).

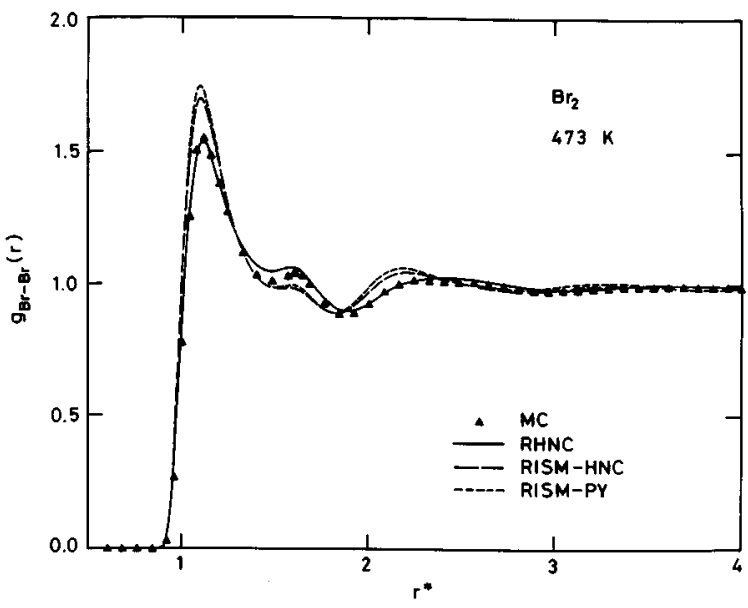

FIG. 11. Same as Fig. 9 but for $T=473 \mathrm{~K}$.

also bear in mind that locating the reference frame on one of the atoms instead of the molecular center, weakens the convergence of the spherical harmonic expansion by increasing the anisotropy of the angular functions. To improve the results including more coefficients is at present a too demanding task, since including coefficients up to $g_{555}$ implies handling an additional set of 21 coefficients, which would add up to the 35 coefficients we are already solving for.

Finally, Figs. 9-12 correspond to $\mathrm{Br}_{2}$ at 293 and $473 \mathrm{~K}$. At $293 \mathrm{~K}$ we have the case with the largest anisotropy and lowest $T^{*}$-higher $\rho^{*}$ we have considered. These stern conditions explain why the success of the RHNC theory is in this case more limited. Comparison with experimental neutron diffraction data also reflects the known inadequacies of twocenter Lennard-Jones potentials to treat liquid $\mathrm{Br}_{2}{ }^{25}$

We can conclude that the use of accurate molecular integral equations to determine the site-site correlation function, and subsequently the atomic structure factor, is a feasible alternative to the simpler but less accurate RISM equations. Although the procedure is still computationally

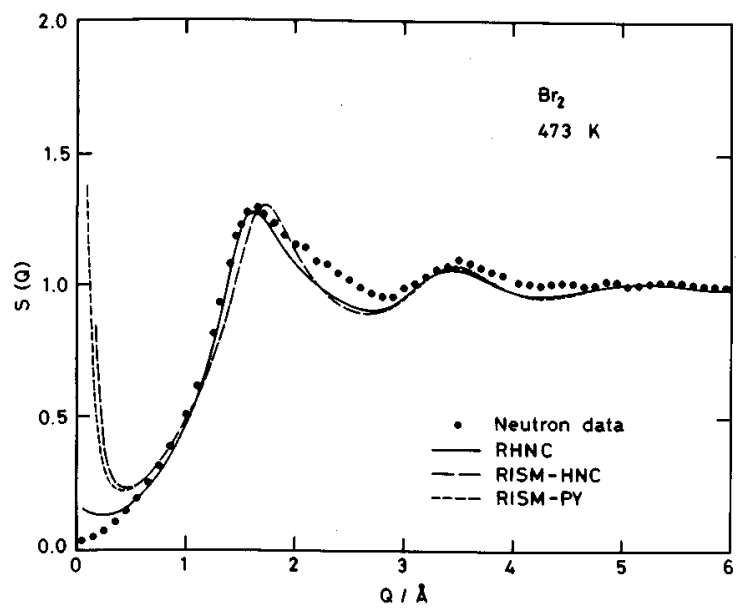

FIG. 12. Same as Fig. 10 but for $T=473 \mathrm{~K}$. Experimental data are neutron diffraction data from Ref. 19. 
expensive, new algorithms are currently under investigation that might reduce drastically the computational demands of the molecular approach. ${ }^{26}$

\section{ACKNOWLEDGMENTS}

We acknowledge the Centro de Supercomputación de Galicia (CESGA) and the Centro Técnico Informático del Consejo Superior de Investigaciones Científicas for generous access to their computing facilities. This research was financially supported by the Spanish Dirección General de Investigación Científica y Técnica (DGICYT) under Grant No. PB91-0110.

${ }^{1}$ F. Lado, S. M. Foiles, and N. W. Ashcroft, Phys. Rev. A, 28, 2374 (1983).

${ }^{2}$ F. Lado, Mol. Phys. 47, 283,299 (1982).

${ }^{3}$ E. Lomba, C. Martín, M. Lombardero, and J. A. Anta, J. Chem. Phys. 96, 6132 (1992).

${ }^{4}$ M. Lombardero, C. Martín, and E, Lomba, J. Chem. Phys. 97, 2724 (1992).

${ }^{5}$ C. Martín, M. Lombardero, and E. Lomba, J. Chem. Phys. 98, 6465 (1993).

${ }^{6}$ C. Martín, E. Lomba, M. Lombardero, F. Lado, and J. S. Høye, J. Chem. Phys. 100, 1599 (1994).

${ }^{7}$ M. Lombardero, C. Martín, and E. Lomba, Mol. Phys. 81, 1313 (1994).

${ }^{8}$ L. Verlet, Mol. Phys. 41, 183 (1980); S. Labik, A. Malijevsky, and W. R. Smith, ibid. 73, 87,495 (1991).
${ }^{9}$ L. J. Lowden and D. Chandler, J. Chem. Phys. 59, 6586 (1973); 61, 5228 (1974); P. T. Cummings, G. P. Morriss, and C. C. Wright, ibid. 86, 1696 (1982).

${ }^{10}$ P. A. Monson, Mol. Phys. 47, 435 (1982).

${ }^{11}$ P. S. Y. Cheung and J. G. Powles, Mol. Phys. 30, 921 (1975).

${ }^{12}$ F. P. Ricci, D. Rocca, and R. Vallauri, Mol. Phys., 60, 1245 (1987).

${ }^{13}$ K. Singer, A. Taylor, and J. V. L. Singer, Mol. Phys. 33, 1757 (1977).

${ }^{14}$ A. H. Narten, E. Johnson, and A. Habenshuss, J. Chem. Phys. 73, 1248 (1980).

${ }^{15}$ J. C. Dore, G. Walford, and D. I. Page, Mol. Phys. 29, 565 (1975).

${ }^{16}$ M. C. Bellisent-Funel, U. Buontempo, C. Petrillo, and F. P. Ricci, Mol. Phys. 71, 256 (1990).

${ }^{17}$ P. Bosi, F. Cilloco, F. P. Ricci, and F. Sacchetti, Mol. Phys. 49, 567 (1983).

${ }^{18}$ A. H. Narten, R. Agrawal, and S. I. Sandler, Mol. Phys. 35, 1077 (1978).

${ }^{19}$ C. Andreani, F. Cilloco, L. Nencini, D. Rocca, and R. N. Sinclair, Mol. Phys. 55, 887 (1985).

${ }^{20}$ J. H. Clarke, J. C. Dore, G. Walford, and R. N. Sinclair, Mol. Phys. 31, 883 (1976).

${ }^{21}$ J. P. Hansen and I. R. McDonald, Theory of Simple Liquids (Academic, London, 1990), Sec. 12.3.

${ }^{22}$ D. Chandler, R. Silbey, and B. Ladanyi, Mol. Phys. 46, 1335 (1982).

${ }^{23}$ F. Hirata and P. J. Rossky, Chem. Phys. Lett. 83, 329 (1981).

${ }^{24}$ R. S. Wu, L. L. Lee, and J. H. Harwell, J. Chem. Phys. 91, 4254 (1989).

${ }^{25}$ P. M. Rodger, A. J. Stone, and D. J. Tildesley, Chem. Phys. Lett. 145, 365 (1988).

${ }^{26}$ J. A. Anta, E. Lomba, C. Martín, M. Lombardero, and F. Lado, Mol. Phys. (in press). 